\title{
MODIFIED HARDY-LITTLEWOOD MAXIMAL OPERATORS ON NONDOUBLING METRIC MEASURE SPACES
}

\author{
Krzysztof Stempak \\ Politechnika Wrocławska, Instytut Matematyki i Informatyki \\ Wyb. Wyspiańskiego 27, 50-370 Wrocław, Poland; Krzysztof.Stempak@pwr.edu.pl
}

\begin{abstract}
We investigate the modified Hardy-Littlewood maximal operators, uncentered $M_{k} f(x)=\sup \frac{1}{\mu(k B)} \int_{B}|f| d \mu$, and centered $M_{k}^{c} f(x)=\sup \frac{1}{\mu(B(x, k r))} \int_{B(x, r)}|f| d \mu, k \geq 1$, in the setting of a general metric measure space $(X, d, \mu)$. By using an enhanced version of the basic covering theorem we prove that $M_{3}$ and $M_{2}^{c}$ are weak type $(1,1)$.
\end{abstract}

\section{Introduction}

Let $(X, d, \mu)$ be a metric measure space, that is let $d$ be a metric and let $\mu$ be a Borel measure on $X$, which we always assume to be finite on bounded sets and nontrivial in the sense that $\mu(X)>0$. Given $r>0$ and $x \in X$ let $B(x, r)=\{y \in$ $X: d(x, y)<r\}$ be the open ball related to $d$ of radius $r$ and with center $x$; if the latter ' $<$ ' is replaced by ' $\leq$ ', then the closed ball is considered. Throughout the paper, if not declared specifically, the term ball and the symbol $B$ will denote either an open or a closed ball. However, if an introduced notion or a declared statement concerns balls, then either only open balls or only closed balls are taken into account. In general, neither the center nor the radius of a ball as a set are uniquely determined. Thus, for a ball $B$ and $k>0$ by $k B$ we always mean the ball concentric with $B$ and of radius $k$ times that of $B$.

For a parameter $k \geq 1$ consider the modified Hardy-Littlewood maximal operator $M_{k}=M_{k, d, \mu}$ acting on a function $f$ by

$$
M_{k} f(x)=\sup _{x \in B \in \mathcal{B}_{0}} \frac{1}{\mu(k B)} \int_{B}|f| d \mu, \quad x \in X .
$$

Here the supremum is taken over all open balls $B \in \mathcal{B}_{0}$ containing $x$, where $\mathcal{B}_{0}=$ $\mathcal{B}_{0}(d, \mu)$ denotes the family of all balls $B$ with the property $\mu(B)>0$. The centered version $M_{k}^{c}=M_{k, d, \mu}^{c}$ is given by

$$
M_{k}^{c} f(x)=\sup _{r>r_{0}(x)} \frac{1}{\mu(B(x, k r))} \int_{B(x, r)}|f| d \mu, \quad x \in X,
$$

where $r_{0}(x)=\inf \{r>0: \mu(B(x, r))>0\}$. For $k=1, M_{1}$ and $M_{1}^{c}$ are the usual, uncentered and centered, Hardy-Littlewood maximal operators on $(X, d, \mu)$; we shall skip the 1 subscript and simply write $M$ and $M^{c}$. Clearly $M_{k}^{c} \leq M_{k}$ (which precisely

doi:10.5186/aasfm.2015.4024

2010 Mathematics Subject Classification: Primary 42B25.

Key words: Metric measure space, non-doubling measure, modified Hardy-Littlewood maximal operator, weak type $(1,1)$.

Research supported by NCN of Poland under grant 2013/09/B/ST1/02057. 
means that $M_{k}^{c} f(x) \leq M_{k} f(x)$ for any function $f$ and every $x \in X$; the analogous agreement is used in similar situations) and for $1 \leq k_{1}<k_{2}$ we have $M_{k_{2}} \leq M_{k_{1}}$, and similarly in the centered case; in particular, $M_{k} \leq M$ and $M_{k}^{c} \leq M^{c}$. If in the above definitions open balls are replaced by the closed ones, then to distinguish the relevant operators from the former ones we write $\bar{M}_{k}$ and $\bar{M}_{k}^{c}$. An easy argument, with a use of the monotone convergence theorem and continuity of $\mu$ from above, shows that $M_{k}=\bar{M}_{k}$ and $M_{k}^{c}=\bar{M}_{k}^{c}$ (see also [11, Lemma 3], where a slightly more general context was considered). Thus, distinguishing between open and closed balls for the modified maximal operators is immaterial.

If $\mu$ is a doubling measure on $X$, that is $\mu(2 B) \lesssim \mu(B)$ uniformly in $B$ (note that this condition implies that $\mu(B)>0$ for every $B$ ), then also $\mu(k B) \lesssim \mu(B)$ uniformly in $B$ for any fixed parameter $k>1$. Thus, for any fixed $k>1$ we have $M_{k} \simeq M$ and $M_{k}^{c} \simeq M$ (this is also because $M^{c} \simeq M$ in the doubling case). Therefore, considering the modified operators $M_{k}$ and $M_{k}^{c}$ is essential only in the non-doubling case. Hence, in what follows, we tacitly assume that $\mu$ is non-doubling.

The operators $M_{k}$ and $M_{k}^{c}$ are important substitutes of the usual Hardy-Littlewood maximal operator and are used frequently in the non-doubling case. See, for instance, [9, p. 469], where $k=3$, and $M_{3}^{c}$ is considered in the setting of separable metric spaces, [13, p. 126], where $k>1$ and $M_{k}$ is considered in the setting of $\mathbf{R}^{n}$ and closed Euclidean cubes with sides parallel to the axes, [7, p. 493], where $k=5$ and $M_{5}$ is considered in the context of geometrically doubling metric spaces, and [10] where $k>1$ and $M_{k}$ and $M_{k}^{c}$ are considered in the setting of separable locally compact metric spaces and a Radon measure.

In the specific case of $\left(\mathbf{R}^{n}, d, \mu\right), n \geq 1$, with the metric $d$ generated by either of the two norms, $\|\cdot\|_{2}$ or $\|\cdot\|_{\infty}$, and an arbitrary Borel measure $\mu$ (finite on bounded sets), the following is known. The centered operator $M^{c}$ is of weak type $(1,1)$ regardless of $n \geq 1$, while the uncentered operator $M_{k}$ is of weak type $(1,1)$ for $k=1$ when $n=1$ and for $k>1$ when $n \geq 2$. In the latter case of $n \geq 2$ the result is sharp since there are examples of measures (the Gaussian measure, for instance) for which $M$ is not of weak type $(1,1)$. Moreover, on the real line, $M_{1}$ is of weak type $(1,1)$ for an arbitrary metric $d$ for which balls are open intervals of the form $(a, b), a<b, a, b \in \mathbf{R} \cup\{ \pm \infty\}$; this is a consequence of a specific covering lemma related to the one-dimensional situation, see [4, p. 31, Lemma 2.6]. On the other hand, in the case of $\mathbf{R}^{n}, n \geq 2$, the assumptions on the metric $d$ cannot be relaxed too much. Aldaz [2] furnished an example of a Borel measure $\mu$ (positive on all balls and of polynomial growth) on the upper half plane $\mathbf{H}=\mathbf{R} \times \mathbf{R}_{+}$equipped with the hyperbolic metric, such that the associated centered maximal operator $M^{c}$ is not of weak type $(1,1)$. Clearly, this example may be readily transferred onto $\mathbf{R}^{2}$.

Throughout the paper a standard notation is applied. By $L^{1}(X)=L^{1}(X, \mu)$ we denote the usual Lebesgue $L^{1}$ space on the measure space $(X, \mu)$. A function $f$ on $X$ is always meant to be Borel measurable, either complex-valued or with values in the extended real number system $\overline{\mathbf{R}}=\mathbf{R} \cup\{ \pm \infty\}$. It may be checked that for any such $f, M_{k} f$ and $M_{k}^{c} f$ are lower semicontinuous, hence Borel measurable (see also [11, Lemma 2], where a slightly more general context was considered). By a local integrability of a function $f$ on $X$ we mean its integrability with respect to the family of all balls; thus $f \in L_{\text {loc }}^{1}(X):=L_{\text {loc }}^{1}(X, d, \mu)$ provided $\int_{B}|f| d \mu<\infty$ for every ball $B$. Note that this notion does not refer to compactness. 


\section{A refinement of the basic covering lemma}

In this section $(X, d)$ is an arbitrary metric space. The following result enhances the 'basic covering theorem', [6, Theorem 1.2].

Theorem 2.1. Let $\tau>3$ be fixed. Every family $\mathcal{F}$ of balls of uniformly bounded radii contains a disjointed family $\mathcal{G}$ such that

$$
\bigcup_{B \in \mathcal{F}} B \subset \bigcup_{B \in \mathcal{G}} \tau B
$$

Simple examples on the real line show that the above result is sharp in the sense that $\tau=3$ is not enough. Indeed, in the case of closed balls, consider the family of closed intervals $\left[0,1-\frac{1}{n}\right]$ and $\left[-1+\frac{1}{n}, 0\right], n \geq 2$, while in the case of open balls, consider the family of open intervals $\left(-2^{-n}, 1-\frac{1}{n}\right)$ and $\left(-1+\frac{1}{n}, 2^{-n}\right), n \geq 3$. However, in the case when the family $\mathcal{F}$ is finite, $\tau=3$ is enough; this is the content of the Vitali-Wiener covering lemma.

The theorem follows from a slightly more general result, which will be also needed in the proof of Theorem 3.1, by taking in Proposition $2.2 \delta=1$ and $\kappa=(\tau-1) / 2$ for a given $\tau>3$.

Proposition 2.2. Let $\kappa>1$ and $\delta>0$ be given. Every family $\mathcal{F}$ of balls of uniformly bounded radii contains a disjointed family $\mathcal{G}$ such that

$$
\bigcup_{B \in \mathcal{F}} \delta B \subset \bigcup_{B \in \mathcal{G}}((1+\delta) \kappa+1) B
$$

Moreover, every ball $B \in \mathcal{F}$ meets a ball from $\mathcal{G}$ with radius at least $\frac{1}{\kappa}$ times that of $B$.

Proof. The proof is a suitable modification of the proof of [6, Theorem 1.2]; to be consistent, we use the analogous notation. Let $\Omega$ denote the set of all disjointed subfamilies $\omega$ of $\mathcal{F}$ with the property: if a ball $B \in \mathcal{F}$ meets a ball $B^{\prime} \in \omega$, then $B$ also meets $B^{\prime \prime} \in \omega$ such that $r\left(B^{\prime \prime}\right) \geq \frac{1}{\kappa} r(B)$. Then $\Omega$ is nontrivial, partially ordered by inclusion, and if $\mathcal{C} \subset \Omega$ is a chain, then also $\omega_{0}=\cup_{\omega \in \mathcal{C}} \omega \in \Omega$. By the Kuratowski-Zorn lemma, there is a maximal element of $\Omega$; denote it by $\mathcal{G}$. We now claim that every $B \in \mathcal{F}$ meets a ball from $\mathcal{G}$. If not, then the collection $\mathcal{A}$ of these balls $B$ that do not meet $\mathcal{G}$ is nonempty and hence we can choose $B_{0} \in \mathcal{A}$ such that $r\left(B_{0}\right) \geq \frac{1}{\kappa} r(B)$ for every $B \in \mathcal{A}$. Consider the disjointed collection $\mathcal{G}^{\prime}=\mathcal{G} \cup\left\{B_{0}\right\}$. By the choice of $B_{0}$, we have $\mathcal{G}^{\prime} \in \Omega$ which contradicts the maximality of $\mathcal{G}$ and the claim follows.

Now, every ball $B \in \mathcal{F}$ meets a ball $B^{\prime} \in \mathcal{G}$ such that $r(B) \leq \kappa r\left(B^{\prime}\right)$. By using the triangle inequality we conclude that $\delta B \subset((1+\delta) \kappa+1) B^{\prime}$, which finishes the proof.

\section{Weak type $(1,1)$ of modified maximal operators}

The following result may be seen as an enhancement of the results of Nazarov, Treil and Volberg [9] (the case of a separable metric space and the centered maximal operator), Tolsa [13] (the case of $X=\mathbf{R}^{n}, d=d_{\infty}$ ), Sawano [10] (the case a separable locally compact metric space and a Radon measure), and Hytönen [7] (the case of a geometrically doubling metric space). See also [12]. 
Theorem 3.1. The maximal operators $M_{3}$ and $M_{2}^{c}$ are of weak type $(1,1)$ with the weak type constants equal to one.

Proof. The proof is rather standard but we include it for completeness. We first treat the uncentered case. Given $f, k \geq 3$ and $\lambda>0$, consider the level set

$$
E_{k, \lambda}(f)=\left\{x \in X: M_{k} f(x)>\lambda\right\} .
$$

We shall prove the estimate

$$
\mu\left(E_{k, \lambda}(f)\right) \leq \frac{1}{\lambda}\|f\|_{1}, \quad f \in L^{1}(X), \quad \lambda>0,
$$

for $k>3$. Since $E_{3, \lambda}(f)=\bigcup_{k>3} E_{k, \lambda}(f)$ and $E_{k, \lambda}(f)$ increases (with respect to inclusion) as $k \rightarrow 3^{+}$, the corresponding estimate for $k=3$ follows by a limiting argument.

Proving (3.1), as usual in such situation, we shall consider the truncated maximal operators $M_{k, R}, R>0$, where $M_{k, R}$ differs from the definition of $M_{k}$, by admitting only the balls of radii $<R$ (convention that $\sup \emptyset=0$ is useful here and later on). Note that $M_{k, R} f$ is lower semicontinuous, hence Borel measurable, for any $f$ (for this fact the reader may consult [11, Lemma 2]).

Thus, since $M_{k, R} \nearrow M_{k}$ as $R \rightarrow \infty$, it suffices to show that

$$
\mu\left(E_{k, \lambda}^{R}(f)\right) \leq \frac{1}{\lambda}\|f\|_{1}, \quad f \in L^{1}(X), \quad \lambda>0,
$$

uniformly in $R \rightarrow \infty$, where $E_{k, \lambda}^{R}(f)$ is defined as $E_{k, \lambda}(f)$, but with $M_{k, R}$ replacing $M_{k}$. The final reduction relies on proving that for a given $R>0$

$$
\mu\left(E_{k, \lambda}^{R}(f) \cap B\left(x_{0}, N\right)\right) \leq \frac{1}{\lambda}\|f\|_{1}, \quad f \in L^{1}(X), \quad \lambda>0,
$$

uniformly in $N \rightarrow \infty$, where $x_{0} \in X$ is a fixed reference point.

Thus, for fixed $k>3, R>1$, and $N>1$, consider

$$
A_{k, R, N}(\lambda, f)=E_{k, \lambda}^{R}(f) \cap B\left(x_{0}, N\right)
$$

and for every $x \in A_{k, R, N}(\lambda, f)$ choose a ball $B_{x}=B\left(y_{x}, r_{x}\right)$ of positive measure and such that $x \in B_{x}, r_{x}<R$ and $\mu\left(k B_{x}\right)<\lambda^{-1} \int_{B_{x}}|f| d \mu$. Note that $B_{x} \subset$ $B\left(x_{0}, N+2 R\right)$. From the covering $\left\{B_{x}\right\}_{x \in A_{k, R, N}(\lambda, f)}$ of $A_{k, R, N}(\lambda, f)$ choose a disjointed subfamily of balls, $\left\{B_{\gamma}\right\}_{\gamma \in \Gamma}, B_{\gamma}=B\left(y_{x(\gamma)}, r_{x(\gamma)}\right)$, as in Theorem 2.1. Since all balls from this subfamily are included in $B\left(x_{0}, N+2 R\right)$, the subfamily is at most countable. Therefore,

$$
\mu\left(A_{k, R, N}(\lambda, f)\right) \leq \mu\left(\bigcup_{\gamma \in \Gamma} k B_{\gamma}\right) \leq \sum_{\gamma \in \Gamma} \mu\left(k B_{\gamma}\right)<\sum_{\gamma \in \Gamma} \lambda^{-1} \int_{B_{\gamma}}|f| d \mu \leq \frac{1}{\lambda}\|f\|_{1} .
$$

This finishes the proof of (3.3) and hence the proof of the statement concerning $M_{3}$.

In the centered case we repeat the above argument, with an appropriate modification of notation, and in the decisive part of the reasoning we argue as follows. For a given $k>2$ and every $x \in A_{k, R, N}^{c}(\lambda, f)$, choose $r_{x}<R$ such that $B_{x}=B\left(x, r_{x}\right)$ is a ball of positive measure such that $\mu\left(k B_{x}\right)<\lambda^{-1} \int_{B_{x}}|f| d \mu$. Writing $k=(1+\delta) \kappa+1$, with some $\delta>0$ and $\kappa>1$, we use Proposition 2.2 to select from the covering 
$\left\{\delta B_{x}\right\}_{x \in A_{k, R, N}^{c}(\lambda, f)}$ of $A_{k, R, N}^{c}(\lambda, f)$ a disjointed subfamily $\left\{B_{\gamma}\right\}_{\gamma \in \Gamma}$, at most countable, with properties as in the proposition. Then

$$
\mu\left(A_{k, R, N}^{c}(\lambda, f)\right) \leq \mu\left(\bigcup_{\gamma \in \Gamma} k B_{\gamma}\right) \leq \sum_{\gamma \in \Gamma} \mu\left(k B_{\gamma}\right)<\sum_{\gamma \in \Gamma} \lambda^{-1} \int_{B_{\gamma}}|f| d \mu \leq \frac{1}{\lambda}\|f\|_{1} .
$$

This finishes the proof of the inequality analogous to (3.3), but with $M_{k}$ replaced by $M_{k}^{c}$ and hence the proof of the statement concerning $M_{k}^{c}$.

Theorem 3.1 is sharp in the sense that, in general, any $k<3$ or any $k<2$ is not enough in the uncentered or in the centered case, respectively, for $M_{k}$ or $M_{k}^{c}$ to be of weak type $(1,1)$. This was shown by Sawano [10]; see [10, Proposition 1.1] for the centered case and comments in the beginning of [10, Section 3] for the uncentered case.

Finally, we comment on a dichotomy property for $M_{k} f, f \in L_{\text {loc }}^{1}(X)$, in the general framework of metric measure spaces, which was well known in the doubling case. In [3] it was discovered that the following dichotomy holds: for any function $f \in B M O\left(\mathbf{R}^{n}\right)$ either $M f \equiv \infty$ or $M f<\infty, x$-a.e. Later on Fiorenca and Krbec, see [5, Theorem 2.2] and remarks following this theorem, observed that the property

$$
\text { "if } M f\left(x_{0}\right)<\infty \text { for some } x_{0} \in \mathbf{R}^{n} \text {, then } M f(x)<\infty, x \text {-a.e." }
$$

should be linked to the bigger class $L_{\text {loc }}^{1}\left(\mathbf{R}^{n}\right)$ rather than only to $B M O\left(\mathbf{R}^{n}\right)$. Aalto and Kinnunen [1] furnished an elegant argument to show that the above-mentioned property holds in the general setting of measure metric spaces with doubling measures.

In the case of an arbitrary (nondoubling) framework of metric measure spaces the corresponding result, expressed in terms of modified Hardy-Littlewood operators, is contained in the following proposition.

Proposition 3.2. Let $f \in L_{\mathrm{loc}}^{1}(X)$ and assume that $M_{k^{\prime}} f\left(x_{0}\right)<\infty$ for a $k^{\prime} \geq 1$ and some $x_{0} \in X$. Then, for $k>2 k^{\prime}+1$ we have $M_{k} f(x)<\infty, \mu$-a.e. $x \in X$.

Proof. Fix $k^{\prime} \geq 1$ and $k>2 k^{\prime}+1$, and let $\delta=\frac{2\left(k^{\prime}+1\right)}{k-\left(2 k^{\prime}+1\right)}$. We claim that for any $f \in L_{\mathrm{loc}}^{1}(X), x_{0} \in X, R>0$, and $\delta>0$ (arbitrary in this moment), it holds

$$
M_{k}\left(f \cdot \chi_{X \backslash B\left(x_{0},(1+\delta) R\right)}\right)(x) \leq M_{k^{\prime}} f\left(x_{0}\right), \quad x \in B\left(x_{0}, R\right) .
$$

Indeed, with $x_{0} \in X, R>0$ and $\delta>0$, for a fixed $x \in B\left(x_{0}, R\right)$ consider any ball $B(y, r)$ such that $x \in B(y, r)$ and $B(y, r) \cap B\left(x_{0},(1+\delta) R\right)^{c} \neq \emptyset$. This implies that $r>\frac{\delta}{2} R, B(y, r) \subset B\left(x_{0}, 2\left(1+\frac{1}{\delta}\right) r\right)$, and for every $a>0$ we have

$$
B\left(x_{0}, a r\right) \subset B\left(y,\left(a+\frac{2}{\delta}+1\right) r\right) .
$$

Majorizing the mean

$$
\frac{1}{\mu(B(y, k r))} \int_{B(y, r)}|f| \chi_{X \backslash B\left(x_{0},(1+\delta) R\right)} d \mu
$$

we replace the region of integration $B(y, r)$ by $B\left(x_{0}, 2\left(1+\frac{1}{\delta}\right) r\right)$ and $\mu(B(y, k r))$ by $\mu\left(B\left(x_{0},\left(k-\left(1+\frac{2}{\delta}\right)\right) r\right)\right)$; note that $k>1+\frac{2}{\delta}$ for $\delta$ chosen in the beginning of the proof. Then the mean (3.4) is replaced by

$$
\frac{1}{\mu\left(B\left(x_{0}, k^{\prime} r^{\prime}\right)\right)} \int_{B\left(x_{0}, r^{\prime}\right)}|f| d \mu
$$


where $r^{\prime}=2\left(1+\frac{1}{\delta}\right) r$ and the claim follows.

With the claim, using the subadditivity of $M_{k}$, we write

$$
M_{k} f(x) \leq M_{k}\left(f \cdot \chi_{B\left(x_{0},(1+\delta) R\right)}\right)(x)+M_{k}\left(f \cdot \chi_{X \backslash B\left(x_{0},(1+\delta) R\right)}\right)(x)
$$

and note that the first term on the right hand side is, by Theorem 3.1, $\mu$-a.e. $x \in X$ finite, and the second term is finite for every $x \in B\left(x_{0}, R\right)$. Letting $R \rightarrow \infty$ completes the proof.

\section{References}

[1] Aalto, D., and J. Kinnunen: The discrete maximal operator in metric spaces. - J. Anal. Math. 111, 2010, 369-390.

[2] Aldaz, J. M.: An example of a maximal function associated to nondoubling measure. - Publ. Mat. 49, 2005, 453-458.

[3] Bennett, C., R. A. DeVore, and R. Sharpley: Weak- $L^{\infty}$ and BMO. - Ann. of Math. (2) $113,1981,601-611$.

[4] Duonndikoetxea, J.: Fourier analysis. - Grad. Stud. Math. 29, Amer. Math. Soc., Providence, RI, 2001.

[5] Fiorenza, A., and M. Krbec: On the domain and range of the maximal operator. - Nagoya Math. J. 158, 2000, 43-61.

[6] Heinonen, J.: Lectures on analysis on metric spaces. - Universitext, Springer, 2001.

[7] Hytönen, T.: A framework for non-homogeneous analysis on metric spaces and the RBMO space of Tolsa. - Publ. Mat. 54, 2010, 484-504.

[8] Lin, C.-C., K. Stempak, and Y.-S. WANG: Local maximal operators on measure metric spaces. - Publ. Mat. 57, 2013, 239-264.

[9] Nazarov, F., S. Treil, and A. Volberg: Weak type estimates and Cotlar inequalities for Calderón-Zygmund operators on nonhomogeneous spaces. - Int. Math. Res. Not. IMRN 9, 1998, 463-487.

[10] Sawano, Y.: Sharp estimates of the modified Hardy-Littlewood maximal operator on the nonhomogeneous space via covering lemmas. - Hokkaido Math. J. 34, 2005, 435-458.

[11] Stempak, K., and X.-X. TAO: Local Morrey and Campanato spaces on quasi-metric measure spaces. - J. Function Spaces 2014, Art. ID 172486, 2014, 1-15.

[12] Terasawa, Y.: Outer measures and weak type $(1,1)$ estimates of Hardy-Littlewood maximal operators. - J. Inequal. Appl. 2006, Art. ID 15063, 2006, 1-13.

[13] Tolsa, X.: BMO, $H^{1}$, and Calderón-Zygmund operators for non doubling measures. - Math. Ann. 319, 2001, 89-149. 